\title{
Review of: "Oxytocin neurons mediates the effect of social isolation via the VTA circuits"
}

\author{
Yang Qian
}

Potential competing interests: The author(s) declared that no potential competing interests exist.

In this manuscript by Stefano Musardo and colleagues, the authors described that one week of social isolation during adolescence in mice increased social interaction in the direct free-interaction task and induced deficits in social novelty preference and social habituation. By using a combination of pharmacogenetics, whole-cell patch clamp recordings, and behavior, they showed that the increased social interaction after social isolation is the consequence of the increased activity of DA neurons within the VTA and this effect is mediated by increased excitability of oxytocin neurons of the PVN. The increased social interaction was also found during adulthood in mice isolated during adolescence which were accompanied by the expression of GluA2-lacking AMPARs on DA neurons that project to the prefrontal cortex (PFC).

1. The title "mediates" should be "mediate"

2. The data analysis of the three-chamber task all adopted intra-group analysis. Is it possible to use inter-group comparison to more directly reflect the differences between grouped and isolated mice?

3. What is the different information delivered by the experiments of the social novelty test and social habituation test?

4. Figure 1 - supplement 3

To investigate whether behavioral deficits induced by social isolation are dependent on age, the authors conducted 7 days of social isolation in adult mice and found that this resulted in a decrease in social time in the isolated mice, which was opposite to the results of adolescent isolation. What is the mechanism under the differences? Do VTA DA neurons and PVN OT neurons still involved?

\section{Figure 2}

According to the material and methods, putative DA neurons are identified only by their position, morphology, and cell capacitance. Could other methods, such as Ih current analysis and staining, be added to identify Putative DA neurons more accurately?

6. Figure 3

To find upstream of the VTA DA neurons, the authors performed immunofluorescence staining with CFOS and found an increase in PVN CFOS in the isolated mice compared to the grouped mice. How does the experiment be performed? Was there a social stimulus that was introduced to experiment mice before perfusion? Besides, whether the change of the expression of CFOS was observed in other brain regions, such as VTA and mPFC? Since the authors later speculated that PVN OT neurons played a role in social 
deficits induced by social isolation, whether the cFOS expression significantly increased in PVN OT neurons?

7. The authors found that the density of PVN OT neurons increased after adolescent social isolation, but there is no more direct experiment to determine the activity or excitability of OT neurons after social isolation. (Electrophysiological tests were performed on VTA-projecting PVN neurons, but subtypes of PVN neurons were not determined).

8. In Figure 5, the AMPA/NMDA ratio did not change. Why do the authors guess that AMDA receptors have changed? Are there any changes in other subtypes of AMPA receptors?

9. Page 4, line 33-34. Is the term "social behavioural skills" appropriate for mice? And how do the above experiments reflect the changes in "social behavioural skills"?

10. OXT-CRE: HM4DI-LOXP mice were used to inhibit PVN OT neurons, but the OT neurons outside PVN were also inhibited in these mice (eg: SON OT neurons). Therefore, how to confirm that the changes in the characteristics of DA neurons and animal behavior are caused by the specific manipulation of PVN OT neurons?

11. Page 5, Line 8. There is no evidence confirmed that the behavioral changes of animals after social isolation were caused by the changes of VTA DA neurons. Therefore, whether the statement here is accurate? (because the inhibition experiment only performed on OT neurons, but not on VTA DA neurons).

12. Page 6 . Line 28,29 . There is no evidence for "increased activity of oxytocin neurons during social interaction".

13. Page 8, Line 14. On page 3, Line 17, the authors mentioned that stage 1 of the three-chamber task was used to measure the "sociability" of animals, and the experimental results showed no effect. Therefore, whether the term "sociability" was used appropriately to describe the changes in social behavior during adulthood.

14. Page 8, Line 15, "Causally Linked" is not used accurately. There is no direct evidence confirmed that the mPFC-projecting VTA DA neurons play a role in the social deficits induced by social isolation. Because the authors only extensively inhibited the GluA2-lacking AMPARs in VTA, rather than specifically inhibited the GluA2-lacking AMPARs on mPFC-projecting VTA DA neurons. 